\title{
Estudio sobre nivel de independencia y necesidad de recursos de apoyo en una población con enfermedades neuromusculares
}

\author{
Survey on level of independence and need for support resources in a \\ population with neuromuscular disorders
}

\begin{abstract}
Resumen
Objetivos: obtener un perfil sobre capacidad funcional y participación de personas con Enfermedades Neuromusculares (ENM), y determinar los recursos de apoyo necesarios para mejorar o mantener su grado de independencia. Metodología: estudio transversal con 24 personas con ENM. En la evaluación se emplearon la Medida de Independencia Funcional (FIM), un checklist de accesibilidad de la vivienda y un cuestionario específico. Resultados: muestra formada por catorce mujeres $(58,3 \%)$ y 10 hombres $(41,7 \%)$. Se obtuvo una media de $61,7(\mathrm{DE}=17,2)$ para la parte motora de FIM sobre 91, indicando un grado moderado de dependencia. El producto de apoyo (PA) más frecuentemente empleado fue la silla de ruedas $(70,8 \%)$. La mayoría de las viviendas $(87,5 \%)$ presentaban barreras arquitectónicas. Conclusiones: la evaluación concisa sobre necesidades de independencia de personas con ENM es fundamental para prescribir los recursos más adecuados para satisfacerlas. Este procedimiento se debe implementar en programas asistenciales, incluyendo la atención al cuidador.
\end{abstract}

\section{Palabras clave}

Enfermedades neuromusculares, actividades de la vida diaria, productos de apoyo, independencia, terapia ocupacional.

\begin{abstract}
Objectives: to obtain a profile on the functional capacity and participation of people with Neuromuscular Disorders (NMD), and to determine the support resources required to improve or maintain their degree of independence. Methods: cross-sectional study with 24 persons with NMD. The Functional Independence Measure (FIM), the checklist of a home's accessibility level and specific questionnaire were administered. Results: sample formed by fourteen women (58.3\%) and 10 men (41.7\%). A mean of $61.7(\mathrm{SD}=17.2)$ was obtained for FIM Motor, over 91, indicating a moderate level of dependence. The assistive technology (AT) most frequently used was the wheelchair $(70.8 \%)$. The architectural barriers were detected in the majority of the users' homes $(87.5 \%)$. Conclusions: a concise assessment of the independence needs of people with NMD and prescription of the most adequate resources to meet them is required. This procedure should be implemented in health-care programs, including care to caregiver.
\end{abstract}

\section{Keywords}

Neuromuscular disorders, activities of daily life, assistive technology, independence, occupational therapy.

\author{
Thais Pousada García
}

<tpousada@udc.es>

Universidad de La Coruña. España

Javier Pereira Loureiro

<javierp@udc.es>

Universidad de La Coruña. España

\section{Betania Groba González}

<bgroba@udc.es>

Universidad de La Coruña. España

\section{Laura Nieto-Riveiro \\ <laura.nieto@udc.es>}

Universidad de La Coruña. España

Alejandro Pazos Sierra

<apazos@udc.es>

Universidad de La Coruña. España

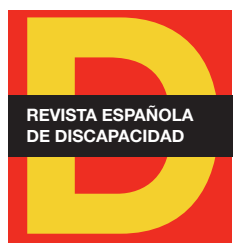

Para citar:

Pousada, T. et al. (2018): "Estudio sobre nivel de independencia y necesidad de recursos de apoyo en una población con enfermedades neuromusculares". Revista Española de Discapacidad, 6 (II): 131-147.

Doi: <https://doi.org/10.5569/23405104.06.02.07>

Fecha de recepción: 19-07-2017 Fecha de aceptación: 08-11-2018 


\section{Introducción}

Las enfermedades neuromusculares (ENM) constituyen un grupo heterogéneo de patologías, que incluyen enfermedades de la motoneurona, alternaciones de los nervios motores o afectación del sistema nervioso periférico (Carter, 2009; Cup et al. , 2007a). A pesar de su gran variabilidad, los síntomas más frecuentemente detectados son la debilidad muscular, la fatiga y la dificultad de ejercicio físico, que implican una reducción de su actividad funcional y un aumento del sedentarismo (Cup et al., 2007a; Cup et al., 2007b; McDonald, 2002; Pieterse et al., 2008).

Dicha condición produce una importante disminución de las capacidades físicas de los pacientes, afectando gravemente a su movilidad, a su capacidad de marcha, y grado de independencia para el desarrollo de las actividades de la vida diaria (AVD) (Natterlund y Ahlström, 2001).

El aumento progresivo de la debilidad muscular es diferente en cada individuo y para cada diagnóstico. Sin embargo, en mayor o menor medida, este síntoma puede afectar negativamente al desarrollo de las actividades que requieren una destreza motora fina y la realización de movimientos contra gravedad, así como aquéllas que implican desplazamientos (Boström y Ahlström, 2004; Boström et al., 2005). Por tanto, debido a esta evolución progresiva, la persona puede requerir mayor ayuda de un cuidador, de adaptaciones del entorno y/o productos de apoyo (PA) para el desarrollo de diferentes actividades (Ahlström y Sjöden, 1996; Duger et al., 2003; McDonald, 2002; Stein y Cassell, 2007).

No cabe duda de la importancia del tratamiento físico como un aspecto básico del programa rehabilitador, pero también es necesario contemplar los recursos de apoyo como factores importantes para el mantenimiento de la independencia en personas con ENM (Stuberg, 2001).

En la literatura se han documentado diferentes estudios sobre la independencia funcional o la eficacia de los programas de rehabilitación en esta población (Boström et al., 2005; Jedeloo et al., 2002; Jutai et al., 2000; Louise-Bender et al., 2002; Natterlund y Ahlström, 2001), pero en su mayoría no han destacado la importancia de las adaptaciones, PA o asistencia personal, como factores facilitadores para la independencia de las personas afectadas. Por este motivo, se considera necesario el desarrollo de un estudio comprensivo sobre la influencia de diferentes tipos de apoyo que pueden producir mejoras en la calidad de vida de las personas con ENM, y con ello, contribuir a la reducción de costes de atención sanitaria y mejorar la eficiencia del sistema sociosanitario.

En base a la Clasificación Internacional del Funcionamiento, Discapacidad y Salud (CIF) (World Health Organization, 2001), que ha identificado y definido a los "factores ambientales", se indica que dichos factores, como los "productos y tecnología" o los "apoyos y relaciones", pueden facilitar o restringir la capacidad de desempeño y participación en diversas actividades. Por tanto, se asume que las adaptaciones de la vivienda y el entorno, así como la selección y entrenamiento del uso de productos de apoyo, son relevantes como factores facilitadores (Duger et al., 2003). Además se destaca que, a pesar del elevado grado de dependencia que puede tener la persona con una ENM progresiva, la mayoría permanecen en sus hogares, gracias a la labor y ayuda de los llamados "cuidadores informales familiares" (Boyer et al., 2006a). 
Los objetivos planteados fueron: (1) obtener un perfil real y completo de la capacidad funcional y participación de las personas con ENM, en diferentes actividades; (2) determinar los recursos de apoyo (productos de apoyo, adaptaciones y/o cuidador) requeridos para mejorar o mantener su grado de independencia.

\section{Metodología}

\subsection{Diseño}

Se empleó un diseño transversal y descriptivo, a fin de determinar la prevalencia de las variables de interés.

Dado que no existe un registro oficial y completo sobre la incidencia y prevalencia de estas enfermedades en Galicia, ni a nivel nacional, el trabajo se ha desarrollado en el ámbito de una entidad sin fines de lucro, de carácter autonómico. Se ha contado con su colaboración, por ser la única fuente que aglutina, de forma global, al mayor número de personas con esta condición en la región.

Este trabajo respeta los criterios y consideraciones del Comité de Ética de Investigación de Galicia. Todos los participantes otorgaron su consentimiento informado a involucrarse en el estudio.

\subsection{Población y selección de participantes}

La selección se realizó a partir de la base de datos de la entidad. Los criterios para la inclusión fueron: (1) diagnóstico probable o confirmado de una ENM, según hallazgos clínicos; (2) residencia en la provincia de A Coruña; (3) edad mayor de 7 años'; (4) residencia en una vivienda, no en un centro o residencia; y (5) tener un grado moderado de discapacidad (mínimo $45 \%$, según el baremo de evaluación del Grado de Discapacidad, Real Decreto 1979/1999).

De la base de datos de la entidad, en A Coruña, residían 72 personas con ENM. Tras la aplicación de los criterios de inclusión, el número se redujo a 45. La principal razón de esta disminución fue la ausencia del cumplimiento del grado mínimo de discapacidad. De los 45 posibles participantes, 15 de ellos no manifestaron interés en involucrarse en el estudio, y otros 6 no dieron respuesta tras el envío de la información. La muestra final la formaron 24 personas.

\subsection{Variables e instrumentos de medida}

Las variables estudiadas se dividieron en tres categorías: (a) características demográficas y clínicas; (b) capacidades para la actividad y participación; (c) factores ambientales. Para la recogida de la información se utilizaron varias herramientas:

1. Se estableció esta edad mínima ya que la principal herramienta de evaluación empleada, la FIM, está diseñada para ser aplicada en mayores de 7 años. 
- Medida de la Independencia Funcional - Functional Independence Measure (FIM): esta escala fue diseñada para determinar el nivel de independencia en la realización de las actividades de la vida diaria (Uniform Data System for Medical Rehabilitation, 1997). El cuestionario incluye 18 ítems, aglutinados en 6 áreas funcionales: autocuidado, control de esfínteres, transferencias, locomoción, comunicación y aspectos cognitivo-sociales. A su vez, estas áreas se agrupan en dos dominios: Motor (13 ítems) y Cognitivo (5 ítems). Cada ítem se puntúa mediante una escala de Likert de 7 puntos, siendo 1 la necesidad de ayuda total y 7 la independencia completa sin apoyos (Uniform Data System for Medical Rehabilitation, 1997). La validez, fiabilidad y sensibilidad de esta herramienta han sido verificados (Dodds et al., 1993; Linacre et al., 1994; Sharrack et al., 1999). La FIM ha sido traducida y validada a su versión española (Rodríguez, 1997), la que se ha empleado en este estudio, mediante entrevista y observación del desarrollo de las AVD.

- Clasificación nivel de deambulación de Hoffer: esta herramienta ha sido empleada no como una valoración funcional de la marcha, sino como método de categorización que permite determinar el nivel de deambulación (o tipo de marcha) de los participantes (Hoffer et al., 1973). En sus orígenes, la clasificación de Hoffer fue empleada para valorar el desplazamiento en personas con mielomelingocele, pero actualmente, es aplicable a diferentes patologías con un componente motor, entre ellas las ENM (Pousada et al., 2014). Esta clasificación, según su terminología original, permitió categorizar a los pacientes en dos grupos, según su tipo de marcha: no ambulantes (incluyendo las categorías de "no deambulación" y "deambulación terapéutica"), y ambulantes funcionales (incluyendo aquellos que presentaba una "deambulación dentro de la vivienda" y/o "en la comunidad") (Hoffer et al., 1973).

- Checklist del nivel de accesibilidad en la vivienda: este registro fue desarrollado por el Centro Estatal de Autonomía personal y Ayudas Técnicas (CEAPAT)2, perteneciente al IMSERSO. El checklist ayuda al profesional a evaluar todos los ítems y factores importantes de la vivienda, a fin de mejorar las características de accesibilidad. La herramienta se divide en 5 secciones, permitiendo valorar el entorno del edificio (parking y entrada principal), espacios comunes del edificio (puertas, pasillos, escaleras, ascensor), entrada principal a la vivienda (puerta y pasillos), características generales de la vivienda (espacios de giro, alturas de interruptores, accesos, escaleras...) y del baño (medidas de sanitarios, espacios, alturas...). Para la aplicación del instrumento y registrar, por tanto, las características del espacio físico, se realizó una visita a la vivienda de todos los participantes.

- Cuestionario propio: para la recolección de datos sobre las características demográficas, clínicas y los aspectos relacionados con el desempeño y participación de la persona en áreas como la educación, trabajo, ocio y participación social, y en su comunidad, se desarrolló un cuestionario específico por parte del grupo de investigación. Como tal, y al ser elaborado específicamente para el presente estudio, sus propiedades psicométricas no han sido cotejadas. Sin embargo, antes de su aplicación definitiva, se realizó un testeo previo con tres personas que cumplían todos los criterios, excepto el de residir en $\mathrm{A}$ Coruña. Esta prueba anterior permitió determinar la idoneidad de las preguntas y concretar las opciones de respuesta en el cuestionario. Esta herramienta fue aplicada en el contexto de una entrevista con el participante y, en caso de que no pudiera responder adecuadamente (por ejemplo, en los menores de edad), se incluía en la misma al cuidador.

Todos los datos fueron recogidos y registrados por el mismo investigador.

2. Véase: http://www.ceapat.es/ceapat_01/cat_apo/index.htm 


\subsection{Procedimiento}

La intervención consistió en la elaboración de un plan para cada participante, desarrollado por un terapeuta ocupacional, que incluía dos fases:

- Evaluación: se realizó una entrevista con cada participante y su cuidador (en los casos necesarios) para la recogida de datos, empleando la FIM y el cuestionario específico. Las características arquitectónicas de la vivienda y su nivel de accesibilidad se determinaron con el checklist. A través de esta evaluación, se obtuvo un perfil ocupacional de los participantes.

- Determinación de recursos de apoyo requeridos (asesoramiento): en base a la información recogida sobre necesidades y requerimientos para mejorar la independencia de las personas afectadas, se realizó un asesoramiento sobre los recursos de apoyo más adecuados a dichas circunstancias individuales. Para cada participante se elaboró un informe de recomendaciones, contemplando tres aspectos: (1) adaptaciones del hogar; (2) productos de apoyo y (3) asistente personal o cuidador. Evidentemente, en este proceso, se tuvieron en cuenta los requerimientos y prioridades de la persona y, por extensión, de su familia. El informe fue elaborado por el mismo terapeuta ocupacional investigador que realizó la recogida de los datos, y discutido con los otros miembros del equipo.

\subsection{Análisis de datos}

Las variables cuantitativas se expresan con su media (DE), mediana y rango. Las variables cualitativas se expresan con sus frecuencias absolutas y porcentajes.

Se emplearon técnicas no paramétricas ya que se asume que la muestra no responde a la distribución normal, tras la aplicación de la prueba Kolmogorov-Smirnov. El nivel de significación fue establecido en $p \leq$ 0.05. La prueba U de Mann Whitney fue empleada para comparar las diferencias de medias de diferentes variables, según el tipo de deambulación. Para establecer comparaciones de medias entre variables policotómicas se aplicó la prueba Kruskal-Wallis. La posible asociación entre las variables cualitativas se valoró utilizando $X^{2}$, mientras que se empleó la prueba de correlación de Spearman para determinar la relación entre variables cuantitativas.

Los datos fueron procesados con el paquete estadístico SPSS v.22 para Windows.

\section{Resultados}

\subsection{Perfil de los participantes}

La muestra estuvo formada por 24 personas con ENM, catorce mujeres (58.3\%) y 10 hombres (41.7\%). La edad media fue de 28.4 años (rango 7-54, DE=14.97). Solo una persona se encontraba trabajando, mientras que el motivo de que el $37.8 \%$ participantes no tuviera empleo era porque habían recibido la calificación legal de incapacidad laboral total o absoluta. La educación secundaria fue el nivel de estudios más alto al- 
canzado por la mayoría de la muestra (62,5\%). Solo una persona vivía sola, ya que el resto de participantes residían en su vivienda con familiares.

El diagnóstico más frecuente fue Distrofia Muscular de Cinturas (25\%), mientras que la media del grado de discapacidad fue del $71.21 \%$ (DE = 18.2). Solo seis personas tenían una marcha funcional, mientras que el resto $(75 \%)$, no presentaban deambulación o esta era muy deficitaria. Todos los datos demográficos y clínicos se muestran en la Tabla 1, mientras que la Tabla 2 ofrece la información referida al conjunto de actividades realizadas por los participantes y su nivel de independencia.

\begin{tabular}{|c|c|c|c|}
\hline \multicolumn{2}{|l|}{ Características Demográficas } & \multirow{2}{*}{$\begin{array}{l}\mathbf{N} \\
10 \\
14 \\
\end{array}$} & \multirow{2}{*}{$\begin{array}{c}\% \\
41.7 \\
58.3\end{array}$} \\
\hline Sexo: & $\begin{array}{l}\text { Hombre } \\
\text { Mujer }\end{array}$ & & \\
\hline Nivel de estudios: & $\begin{array}{l}\text { Educación primaria o inferior } \\
\text { Educación secundaria } \\
\text { Educación superior (Universidad) }\end{array}$ & $\begin{array}{c}5 \\
15 \\
4 \\
\end{array}$ & $\begin{array}{l}20.8 \\
62.5 \\
16.7\end{array}$ \\
\hline Situación laboral: & $\begin{array}{l}\text { Trabajador } \\
\text { En situación de desempleo } \\
\text { Amo/a de casa } \\
\text { Estudiante } \\
\text { Jubilado } \\
\text { En situación de incapacidad laboral }\end{array}$ & $\begin{array}{l}1 \\
2 \\
3 \\
9 \\
0 \\
9\end{array}$ & $\begin{array}{c}4.2 \\
8.3 \\
12.5 \\
37.5 \\
0 \\
37.5\end{array}$ \\
\hline Núcleo de convivencia: & $\begin{array}{l}\text { Solo } \\
\text { Con padres } \\
\text { Con pareja } \\
\text { Con pareja e hijos }\end{array}$ & $\begin{array}{c}1 \\
18 \\
1 \\
4 \\
\end{array}$ & $\begin{array}{c}4.2 \\
75 \\
4.2 \\
16.7\end{array}$ \\
\hline Grado de discapacidad: & $\begin{array}{l}\text { Moderado: } 45<\text { nivel }>65 \% \\
\text { Alto }>65 \%\end{array}$ & $\begin{array}{l}10 \\
14 \\
\end{array}$ & $\begin{array}{l}41.7 \\
58.3\end{array}$ \\
\hline Diagnóstico principal: & $\begin{array}{l}\text { Distrofia muscular de cinturas } \\
\text { Distrofia muscular de Becker } \\
\text { Atrofia muscular espinal } \\
\text { Distrofia muscular de Duchenne } \\
\text { Distrofia miotónica de Steinert } \\
\text { Distrofia muscular facioescapulohumeral } \\
\text { DM congénita de Ulrich } \\
\text { Distrofia muscular congénita } \\
\text { Artrogriposis }\end{array}$ & $\begin{array}{l}6 \\
4 \\
4 \\
2 \\
2 \\
2 \\
1 \\
1 \\
2 \\
\end{array}$ & $\begin{array}{c}25 \\
16.7 \\
16.7 \\
8.3 \\
8.3 \\
8.3 \\
4.2 \\
4.2 \\
8.3 \\
\end{array}$ \\
\hline $\begin{array}{l}\text { Uso de producto de apoyo para la } \\
\text { marcha: }\end{array}$ & $\begin{array}{l}\text { Deambulación funcional (sin PA) } \\
\text { Muletas } \\
\text { Andador } \\
\text { Silla de ruedas de acompañante } \\
\text { Silla de ruedas manual } \\
\text { Silla de ruedas electrónica }\end{array}$ & $\begin{array}{l}6 \\
3 \\
0 \\
3 \\
3 \\
9\end{array}$ & $\begin{array}{c}25 \\
12.5 \\
0 \\
12.5 \\
12.5 \\
37.5\end{array}$ \\
\hline \multirow{2}{*}{ Nivel de deambulación: } & $\begin{array}{l}\text { Deambulación funcional: } \\
\text { Ambulante en la comunidad } \\
\text { Ambulante en la vivienda }\end{array}$ & $\begin{array}{c}12 \\
9 \\
3\end{array}$ & $\begin{array}{c}50 \\
37.5 \\
12.5\end{array}$ \\
\hline & $\begin{array}{l}\text { No deambulación: } \\
\text { Deambulación terapéutica } \\
\text { Ausencia capacidad deambulación }\end{array}$ & $\begin{array}{c}12 \\
3 \\
9\end{array}$ & $\begin{array}{c}50 \\
12.5 \\
37.5\end{array}$ \\
\hline & & Media & DE \\
\hline $\begin{array}{l}\text { Grado de discapacidad (sobre 100) } \\
\text { Edad }\end{array}$ & & $\begin{array}{l}71.21 \\
28.42\end{array}$ & $\begin{array}{r}18.2 \\
14.97\end{array}$ \\
\hline
\end{tabular}

Fuente: elaboración propia. 


\subsection{Independencia funcional y participación}

Actividades de la vida diaria: se empleó la FIM para determinar el nivel de independencia en AVD. Sus resultados varían en un rango de 56 a 188 (sobre un máximo de 126), media de 96.33 (DE = 17.2) y mediana de 97.5. La puntuación media para la sección FIM Motor fue de 61.7 (DE = 17.2), mientras que para la FIM Cognitiva fue de $33.9(\mathrm{DE}=3.2)$.

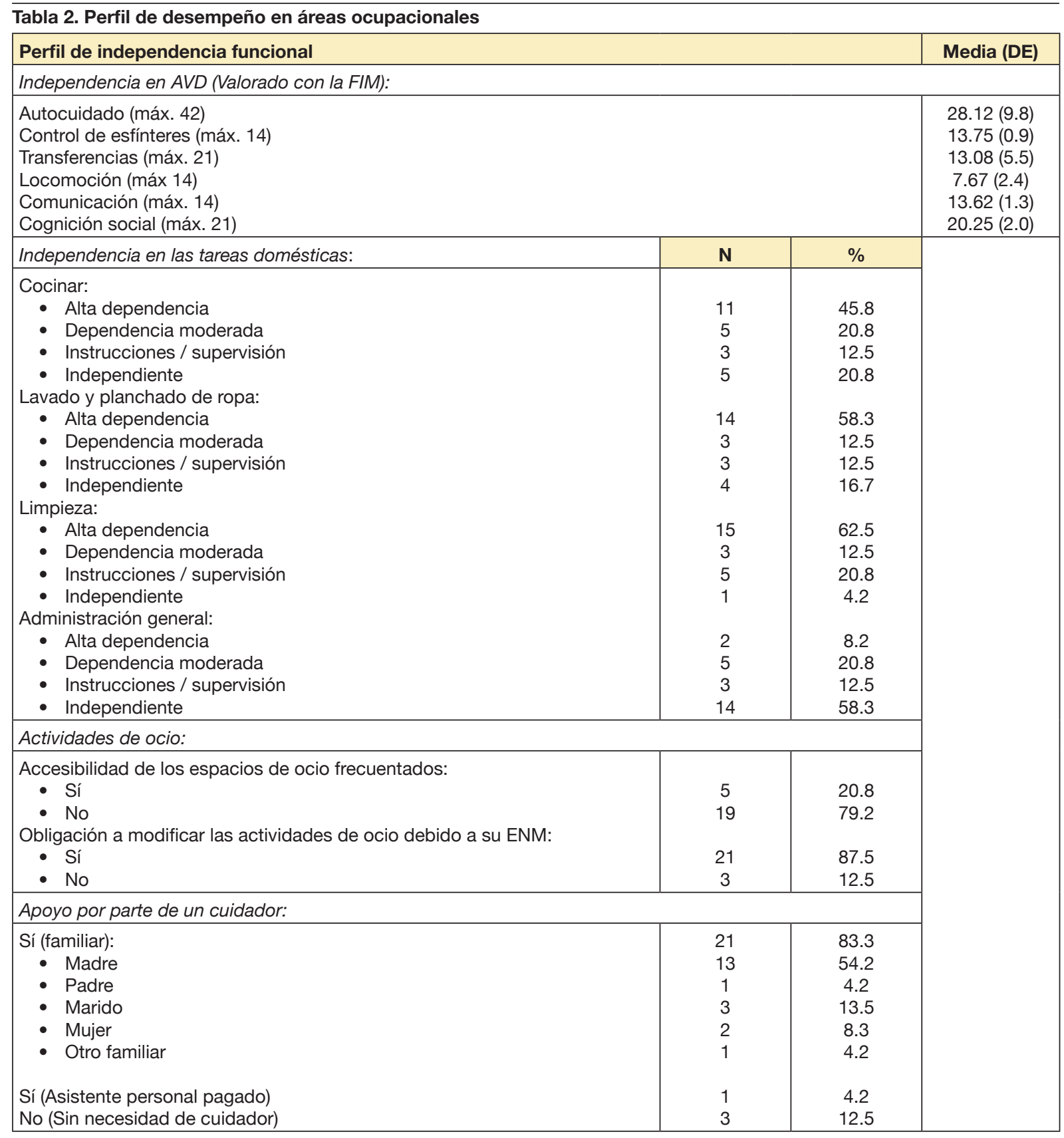




\begin{tabular}{|l|c|c|}
\hline Uso de productos de apoyo: & N & $\%$ \\
\hline Sí (varias opciones posibles de respuesta): & 20 & 83.3 \\
- PA para tratamiento médico & 2 & 8.3 \\
- PA de entrenamiento & 1 & 4.2 \\
- PA para el cuidado y protección & 1 & 4.2 \\
- PA para la movilidad & 16 & 66.7 \\
- Tareas del hogar & 17 & 70.8 \\
- PA de comunicación & 1 & 4.2 \\
- PA para la manipulación & 2 & 8.3 \\
- PA para el ocio & 1 & 4.2 \\
No & 1 & 4.2 \\
\hline Características de accesibilidad de la vivienda: & & \\
\hline Adaptaciones previas en el hogar: & 4 & 16.7 \\
- Sí & & \\
- No & 18 & \\
Presencia de barreras en el hogar (Si): & 6 & 75 \\
- Acceso & & 25 \\
- Entrada principal & 21 & 87.5 \\
- Pasillos & 8 & 33.3 \\
- Comunicación vertical & 10 & 41.7 \\
- Salóna & 13 & 54.2 \\
- Habitación & 4 & 16.7 \\
- Baño & 4 & 16.7 \\
\hline
\end{tabular}

Fuente: elaboración propia.

Además, la puntuación de FIM Motor fue analizada según el tipo de deambulación (según la clasificación de Hoffer) y la edad de la muestra, aplicando la prueba $U$ de Mann Whitney para comprobar si la diferencia de medias era relevante. En este caso, sí se demostró que el tipo de deambulación (funcional o no-deambulación) de los participantes dependía de los resultados de FIM Motor. Es decir, las personas que conservaban un nivel funcional de marcha alcanzaron puntuaciones más elevadas en la sección FIM Motor y, por tanto, un mayor nivel de independencia $(p<0.001)$. Además, se han realizado otros análisis para determinar las relaciones y diferencias en los resultados de la FIM, según diferentes variables, que se muestran en la Tabla 3. 


\begin{tabular}{|c|c|c|c|}
\hline & $\begin{array}{l}\text { FIM Total † } \\
\text { Media (DE) }\end{array}$ & $\begin{array}{c}\text { FIM Motor } ¥ \\
\text { Media (DE) }\end{array}$ & $\begin{array}{c}\text { FIM Cognitivo } \\
\text { Media (DE) }\end{array}$ \\
\hline $\begin{array}{l}\text { Rango de edad: } \\
\qquad 0-18(13) \\
\text { - } 19-50(3) \\
\text { - Más de } 50 \text { años (8) }\end{array}$ & $\begin{array}{l}P=0.840 \\
93(19) \\
94(16) \\
102(16)\end{array}$ & $\begin{array}{l}P=0946 \\
58(19) \\
59(16) \\
69(13)\end{array}$ & $\begin{array}{c}P=0.288 \\
34.67(1,61) \\
35(0) \\
33.12(5,3) \\
\end{array}$ \\
\hline $\begin{array}{l}\text { Nivel de deambulación: } \\
\text { - Ambulantes (12) } \\
\text { - No-ambulantes (12) }\end{array}$ & $\begin{array}{l}P<0.001 \\
107(12) \\
86(15)\end{array}$ & $\begin{array}{l}P<0.001 \\
73(10) \\
50(15)\end{array}$ & $\begin{array}{c}\mathrm{P}=0.655 \\
33.58(4,3) \\
34.17(1,6)\end{array}$ \\
\hline $\begin{array}{l}\text { Uso de } \mathrm{PA}^{*} \text { para la marcha: } \\
\text { - Sí (17) } \\
\text { - No (7) }\end{array}$ & $\begin{array}{l}P<0.005 \\
91(15) \\
110(15)\end{array}$ & $\begin{array}{c}P<0.001 \\
55(16) \\
77(9) \\
\end{array}$ & $\begin{array}{l}P=0.664 \\
34.41(1,4) \\
32.57(5,6)\end{array}$ \\
\hline $\begin{array}{l}\text { Grupo de diagnóstico: } \\
\text { - Distrofias (15) } \\
\text { - Otros (9) }\end{array}$ & $\begin{array}{l}\mathrm{P}=0.215 \\
93(18) \\
101(16)\end{array}$ & $\begin{array}{l}P=0.108 \\
57(18) \\
69(13)\end{array}$ & $\begin{array}{l}P=0.155 \\
34.67(1,3) \\
32.56(4,9)\end{array}$ \\
\hline $\begin{array}{l}\text { Barreras detectadas en hogar: } \\
\text { - Sí (21) } \\
\text { - No (3) }\end{array}$ & $\begin{array}{l}P=0.122 \\
99(15) \\
79(25)\end{array}$ & $\begin{array}{l}P=0.145 \\
64(16) \\
46(23)\end{array}$ & $\begin{array}{l}P=0.680 \\
33.95(3,3) \\
33.33(2,9)\end{array}$ \\
\hline $\begin{array}{l}\text { Adaptaciones previas en hogar: } \\
\text { - Sí (19) } \\
\text { - No (5) } \\
\end{array}$ & $\begin{array}{l}P=0.331 \\
99(15) \\
87(23)\end{array}$ & $\begin{array}{l}P=0.160 \\
65(14) \\
49(23)\end{array}$ & $\begin{array}{c}\mathrm{P}=1.000 \\
33.84(3,5) \\
34(2,24)\end{array}$ \\
\hline $\begin{array}{l}\text { Necesidad de cuidador: } \\
\text { - Sí (21) } \\
\text { - No (3) }\end{array}$ & $\begin{array}{l}P=0.122 \\
112(3) \\
94(17)\end{array}$ & $\begin{array}{l}\mathrm{P}=0.122 \\
77(3) \\
59(17)\end{array}$ & $\begin{array}{l}P=0.561 \\
35(0) \\
33,7(3,4)\end{array}$ \\
\hline
\end{tabular}

*PA: Producto de apoyo.

† Puntuación máxima en $\mathrm{FIM}=126$

‡ Puntuación máxima en FIM Motor $=91$

$\S$ Puntuación máxima en FIM Cognitivo = 35

Fuente: elaboración propia.

Actividades instrumentales de la vida diaria: El cuestionario específico incluía diferentes preguntas para indagar sobre el desempeño de estas tareas. Como se muestra en la Tabla 2, el nivel de independencia de los participantes en el desarrollo de las tareas domésticas fue bajo, en general. La mayoría $(n=18)$ precisaban ayuda por parte de un cuidador en actividades con mayor demanda física, como limpiar o cocinar. Por otra parte, para la ejecución de tareas relacionadas con la administración general, demostraron su independencia en un $58.3 \%$ de los casos $(n=14)$.

Actividades productivas: educación y trabajo: nueve participantes se encontraban estudiando durante la investigación, estando la mayoría $(n=8)$ en un centro educativo ordinario. De este grupo, el $62.5 \%(n=5)$ precisa el apoyo de un cuidador durante las actividades educativas y en 8 casos su puesto escolar había sido adaptado.

En relación con las actividades laborales, solo una persona se encontraba trabajando a tiempo completo. El desplazamiento de este participante a su lugar de trabajo se realizaba en silla de ruedas y su puesto laboral había sido adaptado previamente con pequeños ajustes. 
Ocio y participación social: las actividades de tiempo libre y participación en la vida comunitaria también fueron recogidas, a través del cuestionario específico, por ser áreas importantes para las personas. Al preguntar sobre las actividades de ocio realizadas por los participantes, varias opciones de respuesta eran posibles, siendo las tres más comunes el uso del ordenador $(n=21)$, la lectura $(n=19)$ y salir con los amigos $(n=18)$. Se consultó por aquellas actividades que les gustaría realizar pero no pueden, debido a las barreras del entorno o a su propia condición. En este caso, las tres más deseadas fueron la práctica de deportes $(n=22)$; viajar $(n=20)$ y salir más al aire libre $(n=20)$. La mayoría $(87.5 \%)$ indicó que había tenido que modificar sus actividades de ocio debido a su grado de discapacidad y un $20.8 \%$ determinó que los lugares para la práctica del ocio no están totalmente adaptados.

\subsection{Recursos de apoyo}

A fin de conocer el apoyo físico y personal que precisan los participantes, se analizaron diferentes factores del entorno físico y social (Tabla 2). Solo tres participantes no necesitaban la ayuda de un cuidador (familiar o asistente personal).

Con respecto al uso de PA, la mayoría (83.3\%) empleaba algún dispositivo para el desarrollo de sus actividades cotidianas. El más frecuentemente utilizado para la movilidad personal fue la silla de ruedas $(n=17)$.

El nivel de accesibilidad de la vivienda fue deficiente en casi todos los casos, ya que solo en 3 viviendas no se encontraron barreras. El baño fue la estancia con las peores condiciones de accesibilidad, mientras que en el salón fue donde menos barreras se detectaron. Una amplia mayoría de la muestra (75 \%) realizó adaptaciones previamente en su hogar pero, debido a la prevalente inaccesibilidad, estas fueron insuficientes.

\subsection{Características de marcha y relación con otros factores}

Con respecto al nivel de deambulación, los participantes fueron divididos en dos grupos: los que presentaban una marcha funcional (en la comunidad y en la vivienda, $n=12$ ), y los no-ambulantes (marcha terapéutica o sin deambulación, $n=12$ ); según la clasificación de Hoffer (Hoffer et al., 1973).

La principal diferencia entre ambos grupos fue su nivel de independencia, como se muestran en los resultados de FIM Motor (Tabla 3). Se realizaron diferentes análisis para determinar si existían otros factores condicionantes de dicho nivel de deambulación. Así, se estudiaron las relaciones de esta característica con la edad, grupo diagnóstico, presencia de barreras en la vivienda (y adaptaciones), necesidad de cuidador y uso de PA. Los resultados de este análisis comparativo se muestran en la Tabla 4. Estos muestran que el tipo de diagnóstico (distrofia muscular u otra ENM) puede tener cierta influencia en el nivel de deambulación. Por tanto, las personas con un diagnóstico de DM podrían tener afectada negativamente su capacidad de marcha $(p<0.05)$.

En el análisis de rango de edad, parece ser que la enfermedad afectaría mayormente al nivel de deambulación de los pacientes a edades tempranas, ya que los participantes menores de 18 años presentan la deambulación no funcional más frecuentemente que aquellos mayores de 50 ( $p<0.05)$. Por último, las personas no 
ambulantes utilizaban algún producto de apoyo (tanto para la movilidad como para otras actividades) más frecuentemente que los que presentaban una deambulación funcional.

\subsection{Asesoramiento y determinación de recursos de apoyo}

La segunda fase del estudio contemplaba la elaboración de un informe para la prescripción de productos de apoyo y adaptaciones en la vivienda (productos y tecnología), así como recomendaciones sobre requerimiento del cuidador o asistente personal. Para ello, se consideraron las necesidades a corto y largo plazo de la persona, y la naturaleza progresiva de la enfermedad.

El uso de algún PA fue recomendado para 22 participantes. Para el $87.5 \%$ de la muestra se propuso un plan de adaptación de la vivienda, mientras que la necesidad de cuidador fue considerada para 18 participantes. En 15 de estos, el nivel de atención proporcionado por el cuidador se incrementó, ofreciendo asesoramiento al $50 \%$ de cuidadores sobre higiene postural, ergonomía y sugerencias para realizar las movilizaciones.

\section{Discusión}

La progresión de la debilidad muscular asociada a una ENM implica la necesidad de mayor apoyo por parte de dispositivos o cuidadores, a fin de mantener el nivel de independencia en las AVD (Duger et al., 2003). Este trabajo ha contemplado un análisis global del perfil ocupacional de personas afectadas por una ENM. Además, el estudio proporciona una descripción general sobre los diferentes recursos de apoyo utilizados, y los necesarios, por parte de los participantes, evidenciando la necesidad de considerar su atención interdisciplinar y coordinada desde la atención primaria de salud.

\subsection{Factores para promover la independencia en personas con ENM}

Estudios previos han mostrado que las personas con alguna ENM tienen reducida su capacidad para el desarrollo de las actividades cotidianas, en su mayoría derivados de la debilidad muscular (Ahlström et al., 2006; Carter, 2009; Natterlund y Ahlström, 2001). Nattlerlund y Ahlström identificaron un amplio número de dificultades en las AVD, principalmente en movilidad, cuidado personal y transferencias (Natterlund y Ahlström, 2001). La silla de ruedas, en este estudio, al igual que otros previos (Creek, 2001; Febrer, 2001; McDonald, 2002), es el primer y principal PA utilizado por personas con una enfermedad neurodegenerativa, como las ENM. Por ello, los programas de intervención con esta población deben tener como principal objetivo el mantenimiento de su independencia el mayor tiempo posible (Fujiwara et al., 2009), así como tener en consideración la valoración de aspectos relacionados con su movilidad y capacidad funcional en diferentes entornos, como vivienda o ámbito laboral y la necesidad de prescripción de diversos PA. Así, en el equipo de intervención, el terapeuta ocupacional (TO), tendría un rol importante, especialmente de asesoramiento. 
Las características de accesibilidad son uno de los principales condicionantes en la participación de la población de estudio. El análisis de las actividades de ocio de los participantes muestra un perfil común: realizadas en el hogar, rutinarias y con un componente pasivo. Las actividades deseadas ponen de manifiesto la dificultad que encuentran en espacios públicos, debido a la presencia de barreras. Estos datos son consistentes con los de las características de participación en actividades de ocio de personas con discapacidad referidos en estudios anteriores (INE, 2009; Poveda et al., 1998).

\subsubsection{Los productos de apoyo y adaptaciones como factores ambientales}

Los PA proporcionan a las personas con ENM muchos beneficios en la mejora de su independencia, como el mantenimiento de la movilidad, de su participación en actividades comunitarias y sociales, conservación de fuerza y energía o prevención de caídas y, con ello, contribuyen a la mejora de su calidad de vida. Una integración exitosa de estos dispositivos en su vida diaria requiere la consideración de diferentes aspectos: el significado que la persona atribuye a ese PA, sus expectativas con respecto al mismo, el coste y la forma en que su uso confirma una progresión de su enfermedad, entre otros (Louise-Bender et al., 2002).

Los resultados obtenidos en el presente estudio son difíciles de comparar con los de otros trabajos debido a la escasez de bibliografía en este ámbito concreto. Se evidencia un bajo uso de PA por parte de los participantes, derivado de la falta de conocimiento sobre la existencia de dichos dispositivos, la escasez de información sobre su adquisición y de su elevado coste. Por tanto, parece necesario ofrecer una correcta valoración y completo asesoramiento para atender y satisfacer las necesidades reales de los pacientes. En el caso de las ENM, y durante la prescripción de cualquier recurso de apoyo, los profesionales deben anticipar las futuras necesidades de la persona, atendiendo al tipo de progresión de la enfermedad. Sin embargo, este proceso no es fácil y puede generar estrés en los pacientes y sus familias, ya que la recomendación resalta la naturaleza progresiva de una ENM y que esta revertirá en un aumento de las dificultades a largo plazo (Stuberg, 2001).

La adaptación de la vivienda es prioritaria, a fin de que las personas con ENM puedan mantener su independencia en su entorno inmediato. El propio hogar tiende a ser un "refugio", por lo que, como se ha sugerido en otros trabajos (Evans et al., 2007; Meyers et al., 2002; Richardson y Frank, 2009), la ausencia de accesibilidad es un obstáculo para realizar diferentes maniobras, incluyendo los movimientos con la silla de ruedas. Estas barreras son un factor que limita el uso generalizado de diferentes PA en la vivienda de la persona. En otras palabras, el individuo tiene que adaptarse a las características del entorno físico, contrariamente a lo deseado.

La presencia de barreras en la vivienda de la mayoría de los participantes $(\mathrm{N}=21)$, indica la necesidad de considerar estos factores de forma adecuada, durante el plan de intervención con esta población, a fin de promover su independencia.

\subsubsection{La importancia del apoyo hacia el cuidador}

La presencia de un cuidador familiar contribuye al hecho de que la persona con ENM permanezca en su vivienda (Boyer et al., 2006b). Al igual que los hallazgos del presente trabajo, otros estudios evidenciaron 
que esta figura proporciona un nivel de apoyo elevado, que irá aumentando a medida que la enfermedad del paciente también avanza (Boström y Ahlström, 2004; Natterlund y Ahlström, 2001).

La ayuda en las transferencias (mover a la persona de una superficie a otra) es una de las principales demandas, por lo que el uso de algún PA (por ejemplo, una grúa), podría mejorar y facilitar la labor de cuidados. Para este fin, varios autores han determinado la importancia del seguimiento de la salud del cuidador a fin de identificar problemas que podrían tener efectos adversos en su estado de bienestar y/o en su habilidad para desarrollar las tareas requeridas (Boyer et al., 2006a; Boyer et al., 2006b; Natterlund y Ahlström, 2001).

En este trabajo, se realizó una valoración y asesoramiento sobre las condiciones del cuidador, para apoyar sus tareas e integrarlo activamente en todo el proceso.

La presencia e integración del cuidador en la ejecución de las AVD, movilidad personal y actividades productivas podría tener una influencia positiva en cómo la persona afectada percibe el efecto de los PA.

Así, el proceso de intervención dirigido a personas con ENM debe tener también en cuenta a su familia y cuidador principal, ya que el bienestar y sobrecarga percibida por este, tendrá un efecto en la dispensación de los cuidados y, finalmente, en la calidad de vida del paciente.

\subsection{Limitaciones del estudio y futuras líneas de investigación}

La principal limitación de este trabajo está condicionada por el reducido número de participantes. Esto podría limitar el poder estadístico, a pesar de haber aplicado pruebas no paramétricas. No obstante, proporciona una información de primera mano sobre las necesidades de recursos de apoyo y las preferencias para su selección en personas con ENM.

Otra limitación estaría relacionada con la especificidad del cuestionario propio empleado, lo que podría interferir en su reproductibilidad por otros investigadores. Además, existen ciertas variables que no han sido controladas o medidas, y que podrían constituir factores confundentes, por ejemplo, posibles cambios en la actitud o estado de humor de la persona, consideraciones económicas, recursos sociales o nivel de apoyo emocional recibido.

Otro factor a considerar es que se ha contemplado la elaboración de un informe con recomendaciones y asesoramiento (diseño transversal), por lo que el siguiente paso sería determinar la efectividad real de dicha intervención en una fase de seguimiento posterior.

Por tanto, la necesidad de ampliar la investigación en este ámbito es evidente, y podría estar focalizada hacia un estudio longitudinal que permita establecer el impacto de dichos recursos de apoyo en la vida de las personas con una ENM, así como su influencia final en el nivel de independencia. Además, la incorporación de estándares de medida de resultados y la valoración de constructos relacionados con la calidad de vida, permitirá a clínicos y gerentes tomar medidas adecuadas para la prescripción de los PA más adecuados a las necesidades de esta población. Esto llevaría a un completo emparejamiento entre la persona y sus recursos, contribuyendo con la eficacia y calidad del sistema sanitario. 


\section{Conclusiones}

- Se ha obtenido un perfil sobre la capacidad e independencia funcional de personas con ENM en el desarrollo de diferentes actividades, destacando su bajo grado.

- $\quad$ El nivel de deambulación de los participantes está influido por factores como la edad, el diagnóstico y el uso de PA.

- Los PA y las adaptaciones del entorno son dos recursos fundamentales a considerar en los programas de intervención con esta población para el mantenimiento de su independencia.

- La prescripción de los dispositivos ha de basarse en una evaluación completa de las necesidades del usuario, considerar expectativas y prioridades y proporcionar un completo entrenamiento.

- El apoyo proporcionado por el cuidador es fundamental como factor ambiental facilitador, teniendo en cuenta las necesidades de esta figura en los programas de intervención a fin de mejorar su bienestar y la calidad de los cuidados. 


\section{Referencias bibliográficas}

Ahlström, G. et al. (2006): "A comprehensive rehabilitation programme tailored to the needs of adults with muscular dystrophy". Clinical Rehabilitation, 20 (2): 132-141.

Ahlström, G., y Sjöden, P. O. (1996): "Coping with illness-related problems and quality of life in adult individuals with muscular dystrophy”. Journal of Psychosomatic Research, 41 (4): 365-376. <https://doi.org/10.1016/ S0022-3999(96)00191-2>

Boström, K. et al. (2005): "Sickness impact in people with muscular dystrophy: a longitudinal study over 10 years". Clinical Rehabilitation, 19 (6): 686-694.

Boström, K. y Ahlström, G. (2004): "Living with a chronic deteriorating disease: the trajectory with muscular dystrophy over ten years". Disability and Rehabilitation, 26 (23): 1388-1398.

Boyer, F. et al. (2006a): "Factors relating to career burden for families of persons with muscular dystrophy". Journal of Rehabilitation Medicine, 38: 309-315.

Boyer, F. et al. (2006b): "Family caregivers and hereditary muscular disorders: association between burden, quality of life and mental health". Annales de Readaptation et de Medecine Physique : Revue Scientifique de La Societe Francaise de Reeducation Fonctionnelle de Readaptation et de Medecine Physique, 49 (1): 16-22. <https:// doi.org/10.1016/j.annrmp.2005.08.001>

Carter, G. T. (2009): Rehabilitation management in neuromuscular disease (en línea). <http://emedicine.medscape. com/article/321397-overview>, acceso 5 de diciembre de 2018.

Creek, M. (2001): "Modelling control strategies for foot-and-mouth disease". The Veterinary Record, 149 (10): 311.

Cup, E. H. et al. (2007a): "Exercise Therapy and Other Types of Physical Therapy for Patients With Neuromuscular Diseases: A Systematic Review”. Archives of Physical Medicine and Rehabilitation, 88 (11): 1452-1464. <https://doi.org/10.1016/j.apmr.2007.07.024>

Cup, E. H. et al. (2007b): "Referral of patients with neuromuscular disease to occupational therapy, physical therapy and speech therapy: usual practice versus multidisciplinary advice”. Disability and Rehabilitation, 29 (9): 717-726. <https://doi.org/10.1080/09638280600926702>

Dodds, T. A. et al. (1993): "A validation of the functional independence measurement and its performance among rehabilitation inpatients". Archives of Physical Medicine and Rehabilitation, 74 (5): 531-536.

Duger, T. et al. (2003): "The environmental barriers of children with Muscular Dystrophies and its effect on mother's low back pain”. Disability and Rehabilitation, 25 (20): 1187-1192. <https://doi.org/10.1080/096382803100015 99952>

España. Real Decreto 1971/1999, de 23 de diciembre, de procedimiento para el reconocimiento, declaración y calificación del grado de minusvalía. Boletín Oficial del Estado, 26 de enero de 2000, núm. 22, pp. 3317-3410.

Evans, S. et al. (2007): "Young people's experiences using electric powered indoor - outdoor wheelchairs (EPIOCs): potential for enhancing users' development?". Disability and Rehabilitation, 29 (16): 1281-1294. <https://doi. org/10.1080/09638280600964406> 
Febrer, A. et al. (2001): "Estudio sobre ayudas técnicas en la distrofia muscular de duchenne". Rehabilitación, 35 (1): 20-27 (en línea). <https://doi.org/10.1016/S0048-7120(01)73131-3>, acceso 7 de diciembre de 2018.

Fujiwara, T. et al. (2009): Activities of daily living (ADL) structure of patients with Duchenne muscular dystrophy, including adults. The Keio Journal of Medicine, 58 (4): 223-226.

Hoffer, M. M. et al. (1973): "Functional ambulation in patients with myelomeningocele". The Journal of Bone and Joint surgery. American Volume, 55 (1): 137-148.

Instituto Nacional de Estadística (2009): "Encuesta de discapacidad, autonomía personal y situaciones de dependencia en España, 2008”. Boletín Informativo del INE, 10: 1-12.

Jedeloo, S. et al. (2002): "Client satisfaction with service delivery of assistive technology for outdoor mobility". Disability and Rehabilitation, 24 (10): 550-557. <https://doi.org/10.1080/09638280110108292>

Jutai, J. et al. (2000): "Psychosocial impact of electronic aids to daily living". Assistive Technology: The Official Journal of RESNA, 12 (2): 123-131. <https://doi.org/10.1080/10400435.2000.10132018>

Linacre, J. M. et al. (1994): "The structure and stability of the Functional Independence Measure”. Archives of Physical Medicine and Rehabilitation, 75 (2): 127-132.

Louise-Bender, P. T. et al. (2002): "The shaping of individual meanings assigned to assistive technology: a review of personal factors". Disability and Rehabilitation, 24 (1-3): 5-20.

McDonald, C. (2002): "Physical activity, health impairments and disability in neuromuscular disease". American Journal of Physical Medicine and Rehabilitation, 81: 108-120.

Meyers, A. R. et al. (2002): "Barriers, facilitators, and access for wheelchair users: substantive and methodologic lessons from a pilot study of environmental effects". Social Science \& Medicine (1982), 55 (8): 1435-1446.

Natterlund, B. y Ahlström, G. (2001): "Activities of daily living and quality of life in persons with muscular dystrophy". Journal of Rehabilitation Medicine : Official Journal of the UEMS European Board of Physical and Rehabilitation Medicine, 33 (5): 206-211.

Pieterse, A. J. et al. (2008): "Development of a tool to guide referral of patients with neuromuscular disorders to allied health services. Part two". Disability and Rehabilitation, 30 (11): 855-862. <https://doi. org/10.1080/09638280701403460>

Pousada T. et al. (2015): "Exploring the Psychosocial Impact of Wheelchair and Contextual Factors on Quality of Life of People with Neuromuscular Disorders". Assistive Technology, 27 (4): 246-256. <https://doi.org/10.1080 /10400435.2015.1045996>

Poveda, R. et al. (1998): Problemática de los usuarios de sillas de ruedas en España (vol. 1). Valencia: Instituto de Biomecánica de Valencia.

Richardson, M., y Frank, A. O. (2009): "Electric powered wheelchairs for those with muscular dystrophy: problems of posture, pain and deformity". Disability and rehabilitation.Assistive Technology, 4 (3): 181-188. <https://doi. org/10.1080/17483100802543114>

Rodríguez, L. P. (1997): Medida de la Independencia Funcional (MIF). Guía para la utilización del sistema de datos uniformes para medicina física y de rehabilitación. Madrid: Servicio de Documentación Científica del Grupo FAES.

Sharrack, B. et al. (1999): "The psychometric properties of clinical rating scales used in multiple sclerosis". Brain : A Journal of Neurology, 122 (Pt 1): 141-159. 
Stein, J. y Cassell, J. (2007): An introductory guide for families with a child newly diagnosed with neuromuscular dystrophy or an associated neuromuscular condition (vol. 1). London: Muscular Dystrophy Campain.

Stuberg, W. (2001): "Home accessibility and adaptative equipment in duchenne muscular dystrophy: a case report”. Pediatric Physical Therapy, 13 (4): 169-174.

Uniform Data System for Medical Rehabilitation (1997): Functional Independence Measure, version 5.1. New York: Buffalo General Hospital.

World Health Organization (2001): International classification of functioning, disability and health - ICF (vol. 1). Ginebra: WHO. 